\title{
Algebraic Properties of Toeplitz Operators on the Pluri-harmonic Fock Space
}

\author{
Dieudonne Agbor ${ }^{1}$ \\ ${ }^{1}$ Department of Mathematics, Faculty of Science, University of Buea, Cameroon. \\ Correspondence: Department of Mathematics, Faculty of Science, University of Buea P.O Box 63, Buea, Cameroon. \\ E-mail: dieu_agb@yahoo.co.uk.
}

Received: September 11, 2017 Accepted: October 5, 2017 Online Published: October 26, 2017

doi:10.5539/jmr.v9n6p67～URL: https://doi.org/10.5539/jmr.v9n6p67

\begin{abstract}
We study some algebraic properties of Toeplitz operators with radial and quasi homogeneous symbols on the pluriharmonic Fock space over $\mathbb{C}^{n}$. We determine when the product of two Toeplitz operators with radial symbols is a Toeplitz operator, the zero-product problem for the product of two Toeplitz operators. Next we characterize the commutativity of Toeplitz operators with quasi homogeneous symbols and finally we study finite rank of the product of Toeplitz operators with quasi homogeneous symbols.
\end{abstract}

Keywords: Fock space, Mellin transform, Pluriharmonic Fock space, Toeplitz operators.

\section{Introduction}

Let $n \in \mathbb{N}$ and consider a family $\left\{\mu_{s}\right\}_{s>0}$ of normalized Gaussian measures on $\mathbb{C}^{n}$ defined by

$$
d \mu_{s}(z):=(s \pi)^{-n} \exp \left\{-\frac{|z|^{2}}{s}\right\} d v(z) .
$$

Here $d v$ denotes the usual Lebesgue volume form on $\mathbb{C}^{n} \cong \mathbb{R}^{2 n}$ and we write $|\cdot|$ for the Euclidean norm on $\mathbb{C}^{n}$. The (Segal-Bargmann) Fock space $H_{s}^{2}=H^{2}\left(\mathbb{C}^{n}, \mu_{s}\right)$ consists of all $\mu_{s}$-square integrable entire functions on $\mathbb{C}^{n}$. As is well known, $H_{s}^{2}$ forms a closed subspace of $L_{s}^{2}:=L^{2}\left(\mathbb{C}^{n}, \mu_{s}\right)$ and we write $P^{(s)}: L_{s}^{2} \rightarrow H_{s}^{2}$ for the orthogonal projection. We let $P=P^{(1)}, H^{2}=H_{1}^{2}$. Let $H_{a h}^{2}$ be tha space of all anti-holomorphic functions in $L^{2}=L_{1}^{2}$ and we denote the pluriharmonic Fock space over $\mathbb{C}^{n}$ by $H_{p h}^{2}$. We recall that $H_{p h}^{2}$ consist of all $C^{2}$-functions $f$ in $L^{2}$ such that $\partial^{2} f / \partial z_{j} \partial \bar{z}_{k}=0$ for all $j, k$. It is known that $H_{a h}^{2}$ and $H_{p h}^{2}$ are all closed subspaces of $L^{2}$ and they have reproducing kernels $\bar{K}$ and $K_{p h}$ respectively, where $K$ is the reproducing kernel for $H^{2}$. Moreover there are orthogonal projections $P_{a h}$ and $P_{p h}$ from $L^{2}$ onto $H_{a h}^{2}$ and $H_{p h}^{2}$ respectively. It is shown in (Englis, 2009), that the reproducing kernel for $H_{p h}^{2}$ is given by

$$
K_{p h}(z, w)=K(z, w)+\overline{K(z, w)}-1, \quad z, w \in \mathbb{C}^{n},
$$

and $P_{p h}=P+P_{a h}-P_{\mathbb{C}}$ is the orthogonal projection operator of $L^{2}$ onto $H_{p h}^{2}$.

For a function $f \in L^{\infty}\left(\mathbb{C}^{n}\right)=L^{\infty}$, we define the Toeplitz operator $T_{f}: H_{p h}^{2} \rightarrow H_{p h}^{2}$, with symbol $f$, by

$$
T_{f} g=P_{p h}(f g), \quad g \in H_{p h}^{2} .
$$

For the product problem, on the Hardy space, it was shown in (Brown and Halmos, 1964), that if $f$ and $g$ are bounded functions on the unit circle, then $T_{f} T_{g}$ is a Toeplitz operator if and only if either $\bar{f}$ or $g$ is analytic. In the setting of the Bergman space, the condition that either $\bar{f}$ or $g$ is analytic is still sufficient but it is no longer necessary. This problem becomes more complicated on the Hardy space over the unit sphere and Bergman space over unit ball in $\mathbb{C}^{n}$. In $($ Choe et al., 2007), this problem was resolved for Toeplitz operators with plurihamonic symbols on the Bergman space of the polydisk while (Dong and Zhou, 2009), were able to determine when the product of two radial Toeplitz operators is a Toeplitz operator. For the case of Fock space, things are a lot more different. It was shown in (Coburn, 2007), that if the symbols $f, g$ are either polynomials in $z, \bar{z}$ or the symbols belong to the Bochner algebra, then $T_{f} T_{g}$ is a Toeplitz operator with the symbols given explicitely. Furthermore, in (Bauer, 2009), it is shown that if the symbols $f$ and $g$ belong to the range of the Heat transform at some time $t>0$ then $T_{f} T_{g}$ is a Toeplitz operator. Recently, in (Agbor and Bauer, 2014), this result has been extended to finite products.

The "zero-product "problem for Toeplitz operators has captured people's attentions for a long time, we mention just a few here. For the case of the Hardy space, the problem has been solved in (Aleman and Vukotic, 2009), while an affirmative 
answer was given to the "zero-product "problem in (Axler and $\breve{C} u c \breve{c k o v i c ́, ~ 1991), ~ f o r ~ T o e p l i t z ~ o p e r a t o r s ~ o n ~ t h e ~ B e r g m a n ~}$ space over the unit disc (in the case of operator products of length 2) if the symbols are both bounded harmonic functions. For the Fock space, and for products of length 2, the problem has been solved in (Bauer and Lee, 2011), in the case when one of the symbols is radial.

The commuting problem for Toeplitz operators on the Hardy, Bergman and Fock spaces have generated a lot of research

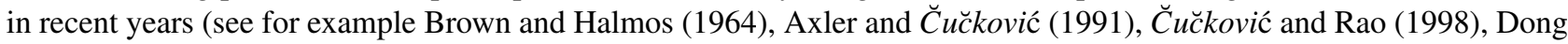
and Zhou (2009), Choe and Lee (1993), Guan et al (2013), Yan and Liu (2013), Bauer and Le (2011), Bauer and Lee (2011), Bauer and Issa (2012) and Bauer et al (2015)). In (Brown and Halmos, 1964), it is shown for the Hardy space on the unit circle that the Toeplitz operators $T_{f}$ and $T_{g}$ commute if and only if either (1) $f$ and $g$ are holomorphic or (2) $f$ and $g$ are anti-holomorphic or (3) one is a linear function of the other. It was shown that the same result holds true in

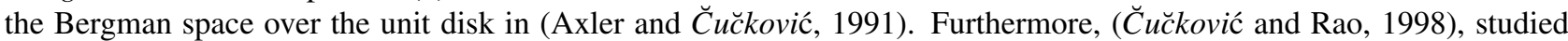
the commutivity of two Toeplitz operators on the Bergman space and described those Toeplitz operators which commute with the Toeplitz operator $T_{e^{i \theta} r^{m}}$ for $p, m \in \mathbb{N}$. Also, (Guan et al., 2013), studied and characterized commuting Toeplitz operator with quasihomogeneous and separately quasihomogeneous symbols on the pluriharmonic Bergman space over the unit ball of $\mathbb{C}^{n}$. For the case of the Fock space, (Bauer and Lee, 2011), showed that if $f$ and $g$ have atmost exponential growth and $f$ is radial and non-constant then $T_{f}$ and $T_{g}$ commute implies that $g$ is radial. In (Bauer et al., 2015), the case when the symbols are pluri-harmonic and satifies some exponential growth condition has been studied.

For the finite rank problem, we note that for the case of a single operator on the Bergman space over domains $\Omega \subset \mathbb{C}^{n}$ this problem had been considered for a long time, and positive answers were given in the case were $\Omega$ is bounded or where $\Omega=\mathbb{C}^{n}$, see for example (Luecking (2008), Rozenblum and Shirokov (2010)). For the case of products of Toeplitz operators and commutators we mention the work in (Yang et al., 2013), on the Pluri-harmonic Bergman space.

Motivated by the results in (Guan et al. (2013), Yang et al. (2013)), on the pluriharmonic Bergman space on the unit ball and the results in (Bauer and Le (2011), Bauer and Lee (2011)), on the Fock space, we study the product of two Toeplitz operators with radial symbols on the pluriharmonic Fock space. We also study the zero-product problem, where we show that the main result in (Bauer and Lee, 2011), also holds in the setting of the pluriharmonic Fock space. Next we consider the commuting problem with quasihomogeneous symbols and finally we consider the finite rank problem for product of two Toeplitz operators with quasihomogeneous symbols and a commutator of Toeplitz operators with such symbols.

The paper is organized as follows: In section 2 we will study some preliminaries. In section 3 we will study the product problem. In section 4 , we consider zero product problem while in section 5, we consider the commuting problem. Finally, in section 6 , we study the finite rank problem.

\section{Preliminaries}

Let $\alpha=\left(\alpha_{1}, \cdots, \alpha_{n}\right) \in \mathbb{N}_{0}^{n}$ and $z=\left(z_{1}, \cdots, z_{n}\right) \in \mathbb{C}^{n}$. We write $|\alpha|=\alpha_{1}+\cdots+\alpha_{n}, \quad \alpha !=\alpha_{1} ! \cdots \alpha_{n}$ ! and $z^{\alpha}=z_{1}^{\alpha_{1}} \cdots z_{n}^{\alpha_{n}}$. For two multi-indexes $\alpha=\left(\alpha_{1}, \cdots, \alpha_{n}\right), \beta=\left(\beta_{1}, \cdots, \beta_{n}\right) \in \mathbb{N}_{0}^{n}$, we denote by $\alpha \gtrsim \beta$ (respectively, $\alpha \lesssim \beta$ ) to imply $\alpha_{i} \geq \beta_{i}$, (respectively, $\alpha_{i} \leq \beta_{i}$ ) for $i=1, \cdots, n$. We write $\alpha \perp \beta$ to imply $\alpha_{1} \beta_{1}+\cdots+\alpha_{n} \beta_{n}=0$. Let $(\alpha-\beta)$ denote $\left(\alpha_{1}-\beta_{1}, \cdots, \alpha_{n}-\beta_{n}\right)$. Moreover, if $\alpha \gtrsim \beta$ then $|\alpha-\beta|=|\alpha|-|\beta|$. For $\alpha \in \mathbb{N}_{0}^{n}$ and $z \in \mathbb{C}^{n}$ we let $e_{\alpha}(z)=\frac{1}{\sqrt{\alpha} !} z^{\alpha}$ then $\left\{e_{\alpha}: \alpha \in \mathbb{N}_{0}^{n}\right\}$ is a basis for the Fock space and $\left\{\bar{e}_{\alpha}: \alpha \in \mathbb{N}_{0}^{n}\right\}$ is a basis for the space $H_{a h}^{2}$. It follows that $\left\{e_{\alpha}: \alpha \in \mathbb{N}_{0}^{n}\right\} \cup\left\{\bar{e}_{\alpha}: \alpha \in \mathbb{N}_{0}^{n}\right\}$ is a basis for the pluri harmonic Fock space $H_{p h}^{2}$.

Now we recall the definition of the symbol space $\operatorname{Sym}_{>0}\left(\mathbb{C}^{n}\right)$ in (Bauer, 2009), together with the construction of a scale of Banach spaces on which the class of Toeplitz operators $T_{f}^{(s)}$ with $f \in \operatorname{Sym}_{>0}\left(\mathbb{C}^{n}\right)$ acts by a "finite order shift". For each $c \in \mathbb{R}$ we set

$$
\mathcal{D}_{c}:=\left\{f: \mathbb{C}^{n} \rightarrow \mathbb{C} \mid f \text { locally integrable and } \exists d>0 \text { s.t. }|f(z)| \leq d e^{c|z|^{2}} \text { a.e }\right\} .
$$

Equipped with the norm $\|f\|_{\mathcal{D}_{c}}:=\left\|e^{-c||^{2}} f\right\|_{\infty}$ the space $\left(\mathcal{D}_{c},\|\cdot\|_{\mathcal{D}_{c}}\right)$ turns into a Banach space. Consider the following strictly increasing sequence

$$
c_{j}(s):=\frac{1}{2 s}-\frac{1}{2 s j+2 s}<\frac{1}{2 s}, \quad j \in \mathbb{N} .
$$

It is easy to check that $\mathcal{D}_{c}$ is continuously embedded into $L^{2}\left(\mathbb{C}^{n}, \mu_{s}\right)$ whenever $c<1 /(2 s)$. Hence we obtain an increasing scale of Banach spaces

$$
L^{\infty}\left(\mathbb{C}^{n}\right)=\mathcal{D}_{c_{0}(s)} \subset \mathcal{D}_{c_{1}(s)} \subset \cdots \subset \mathcal{D}^{s}:=\bigcup_{j \in \mathbb{N}} \mathcal{D}_{c_{j}(s)} \subset L^{2}\left(\mathbb{C}^{n}, \mu_{s}\right) .
$$

Let $k \in \mathbb{N}$, then we write $O_{s}(k)$ for the space of all linear operators $A: \mathcal{D}^{s} \rightarrow \mathcal{D}^{s}$ that continuously map $\mathcal{D}_{c_{j}(s)}$ into $\mathcal{D}_{c_{j+k}(s)}$ for all $j \in \mathbb{N}_{0}$. We say that operators in $O_{s}(k)$ act on the scale (2) by an order shift $k$. The algebra of operators on $\mathcal{D}^{s}$ 
acting on (2) by a finite order shift is given by

$$
\mathcal{L}^{\mathrm{fos}}\left(\mathcal{D}^{s}\right):=\bigcup_{k \in \mathbb{N}_{0}} O_{s}(k)
$$

Lemma 2.1. The orthogonal projection $P_{p h}: L^{2} \rightarrow H_{p h}^{2}$ defines an element in $O(1)=O_{1}(1)$. Moreover for $j \in \mathbb{N}_{0}$ and $g \in \mathcal{D}_{c_{j}}$ we have

$$
\left\|P_{p h} g\right\|_{\mathcal{D}_{c_{j+1}}} \leq C_{j}\|g\|_{\mathcal{D}_{c_{j}}}
$$

Proof It is sufficient to prove (3). Observe that

$$
\left\|P_{p h} g\right\|_{\mathcal{D}_{c_{j+1}}} \leq\|P g\|_{\mathcal{D}_{c_{j+1}}}+\left\|P_{a h} g\right\|_{\mathcal{D}_{c_{j+1}}}+|P g(0)| .
$$

By Lemma 10 of (Bauer, 2009) we have

$$
\|P g\|_{\mathcal{D}_{c_{j+1}}} \leq \frac{\|g\|_{\mathcal{D}_{c_{j}}}}{\left(1-c_{j}\right)^{n}}
$$

A similar computation also shows that

$$
\left\|P_{a h} g\right\|_{\mathcal{D}_{c_{j+1}}} \leq \frac{\|g\|_{\mathcal{D}_{c_{j}}}}{\left(1-c_{j}\right)^{n}}
$$

Also, a direct calculation shows that

$$
\begin{aligned}
|P g(0)| & \leq \int_{\mathbb{C}^{n}}|g(z)| d \mu(z) \\
& \leq\|g\|_{\mathcal{D}_{c_{j}}} \int_{\mathbb{C}^{n}} e^{c_{j}||^{2}} d \mu(z) \leq \frac{\|g\|_{\mathcal{D}_{c_{j}}}}{\left(1-c_{j}\right)^{n}},
\end{aligned}
$$

which gives the desired result.

In order to define Toeplitz operators acting on the scale (2) we restrict ourselves to the following space of (i.g. unbounded) symbols:

$$
\operatorname{Sym}_{>0}\left(\mathbb{C}^{n}\right):=\bigcap_{j=1}^{\infty} \mathcal{D}_{1 / j} .
$$

Clearly, with respect to pointwise multiplication and complex conjugation (5) turns into a $*$-algebra which in particular contains all polynomials in the complex variables $z$ and $\bar{z}$. Furthermore, we remark that for $f \in \operatorname{Sym}_{>0}\left(\mathbb{C}^{n}\right)$ and all $s>0$ the multiplication operator $M_{f}$ defines an element in $O_{s}(1)$. By Lemma 2.1 it follows that

$$
T_{f}=P_{p h} M_{f} \in O(2) \subset \mathcal{L}^{\mathrm{fos}}(\mathcal{D}),
$$

and the restriction of this operator product to $\left(\mathcal{D} \cap H^{2}\right)+\left(\mathcal{D} \cap H_{a h}^{2}\right)$ is the Toeplitz operator with symbol $f$. Since $\mathcal{L}^{\text {fos }}(\mathcal{D})$ is an algebra, all finite products of Toeplitz operators $T_{f}$ with symbols $f \in \operatorname{Sym}_{>0}\left(\mathbb{C}^{n}\right)$ are well-defined and can be interpreted as densely defined (i.g. unbounded) operators on the Hilbert space $H_{p h}^{2}$. Similarly we can consider the spaces $\mathcal{H}_{c}^{h}:=\mathcal{D}_{c} \cap H^{2}\left(\mathbb{C}^{n}, \mu\right)$ and $\mathcal{H}_{c}^{a h}:=\mathcal{D}_{c} \cap H_{a h}^{2}\left(\mathbb{C}^{n}, \mu\right)$ equipped with the norm in $\mathcal{D}_{c}$. We obtain a scale of Banach spaces:

$$
\mathbb{C} \equiv \mathcal{H}_{c_{0}}^{h} \subset \mathcal{H}_{c_{1}}^{h} \subset \cdots \subset \mathcal{H}_{h}:=\bigcup_{j \in \mathbb{N}} \mathcal{H}_{c_{j}}^{h} \subset H^{2}\left(\mathbb{C}^{n}, \mu\right)=H^{2}
$$

and

$$
\mathbb{C} \equiv \mathcal{H}_{c_{0}}^{a h} \subset \mathcal{H}_{c_{1}}^{a h} \subset \cdots \subset \mathcal{H}_{a h}:=\bigcup_{j \in \mathbb{N}} \mathcal{H}_{c_{j}}^{a h} \subset H_{a h}^{2}\left(\mathbb{C}^{n}, \mu\right)=H_{a h}^{2}
$$

Now, we set for each $j \in \mathbb{N}$,

$$
\mathcal{H}_{c_{j}}^{p h}=\mathcal{H}_{c_{j}}^{h} \oplus\left\{\mathcal{H}_{c_{j}}^{a h} \ominus \mathbb{C}\right\} .
$$

Then we obtain the following scale of Banach spaces:

$$
\mathbb{C} \equiv \mathcal{H}_{c_{0}}^{h} \subset \mathcal{H}_{c_{1}}^{p h} \subset \cdots \subset \mathcal{H}_{p h}:=\bigcup_{j \in \mathbb{N}} \mathcal{H}_{c_{j}}^{p h} \subset H_{p h}^{2}\left(\mathbb{C}^{n}, \mu\right)=H_{p h}^{2}
$$


Completely analogous to our definition above we can consider the algebra $\mathcal{L}^{\text {fos }}\left(\mathcal{H}_{p h}\right)$ of all operators acting on (8) by a finite order shift.

A function $f \in \operatorname{Sym}_{>0}\left(\mathbb{C}^{n}\right)$ is said to be radial if $f(U z)=f(z)$ for any unitary operator $U$ of $\mathbb{C}^{n}, f$ is separately radial if and only if $f(U z)=f(z)$ for any unitary transformation $U$ of $\mathbb{C}^{n}$ with a diagonal matrix. Thus a radial function has the form $f(z)=f(|z|)$ while a separately radial if $f(z)=f\left(\left|z_{1}\right|, \cdots,\left|z_{n}\right|\right)$.

Let $p, s \in \mathbb{N}^{n}$ with $p \perp s$ and $f \in \operatorname{Sym}_{>0}\left(\mathbb{C}^{n}\right)$. Then $f$ is called quasihomogeneous of degree $(p, s)$ if there is a radial function $\phi \in \operatorname{Sym}_{>0}\left(\mathbb{C}^{n}\right)$ such that $f(z)=f(r \xi)=\xi^{p} \bar{\xi}^{s} \phi(r)$, for any $\xi \in \mathbb{S}_{n}$, where $r=|z|$.

We recall the definition of the Mellin Transform: Given a function $\psi$ on the half line $(0, \infty)$ the Mellin transform $M[\psi](z)$ of the complex parameter $z$ is defined by

$$
M[\psi](z)=\int_{0}^{\infty} \psi(t) t^{z-1} d t
$$

whenever the improper integral exists. Also, each $M[\psi]$ is complex analytic on a strip in the complex plane parallel to the imaginary axis. Moreover, the Mellin transform is injective. We also recall that if $f, g: \mathbb{R}^{+} \rightarrow \mathbb{C}$ and $x>0$ the Mellin convolution is defined by

$$
(f * g)(x)=\int_{0}^{\infty} f(y) g\left(\frac{x}{y}\right) d y
$$

The Mellin convolution theorem implies $M[f * g]=M[f] \cdot M[g]$ on a certain strip in the complex plane parallel to the imaginary axis.

Let $\mathcal{A}$ denote the class of complex valued functions $u$ for which there exist constants $C, c, \eta, \rho>0$ such that $|u(x)| \leq c x^{-\rho}$ for all $x \in(0,1]$ and $|u(x)| \leq C x^{\eta}$ for $x \in[1, \infty)$.

The following is Proposition 4.8 of (Bauer and Lee, 2011).

Proposition 2.2. Let $u, v \in \mathcal{A}$ and let $f_{u}(x)=u(x) e^{-x^{2}}$ and $f_{v}(x)=v(x) e^{-x^{2}}$. Then $f_{u} * f_{v}$ exists on $\mathbb{R}^{+}$and there is an $h \in \mathcal{A}$ such that $\left(f_{u} * f_{v}\right)(x)=h(x) e^{-x}$.

Furthermore, if suppv $\subset[0,1]$ then $\left(f_{u} * v\right)(x)=h(x) e^{-x^{2}}$ for some $h \in \mathcal{A}$ and all $x \in \mathbb{R}^{+}$.

The following lemma replaces the Blaschke condition in the unit disc and this is Proposition 4.11 of (Bauer and Lee, 2011).

Lemma 2.3. Suppose that $u \in \mathcal{A}$ and $a \in(0,2]$. If there is an $m \in \mathbb{N}$ such that

$$
M\left[u e^{-x}\right](a k+1)=\int_{0}^{\infty} u(x) x^{a k} e^{-x} d x=0
$$

for all $k \geq m$ then $u=0$ a.e. on $\mathbb{R}^{+}$.

We recall that for a suitable holomorphic function $\Psi(z)$ on the right half-plane $\operatorname{Rez}>\delta$, the inverse Mellin transform is given by

$$
\left[M^{-1} \Psi\right](x)=\frac{1}{2 \pi i} \int_{c-i \infty}^{c+i \infty} x^{-s} \Psi(s) d s
$$

Let $\Psi(z):=\prod_{i=1}^{k}(z+i)^{-1}$ for $k \in \mathbb{N}$ and $\operatorname{Re}(z)>-1$. Then we have the following which is Lemma 4.5 of (Bauer and Lee, 2011).

Lemma 2.4. The inverse Mellin transform $m_{k}(x):=\left[M^{-1} \Psi\right](x)$ has support in $[0,1]$. Moreover, $m_{k}(x)=O\left(x^{\alpha}\right)$ for all $\alpha<1$ as $x \rightarrow 0$.

Let $\mathbb{K}=\{z \in \mathbb{C}: \operatorname{Re}(z)>0\}$ the right half of the complex plane. Let $f \in \mathcal{D}_{c}$, for $c<1$, then we define

$$
\mathcal{F}[f](z)=\int_{\mathbb{C}^{n}} f(w)\left|w_{1}\right|^{2 z_{1}} \cdots\left|w_{n}\right|^{2 z_{n}} d \mu(w)
$$

for any $z=\left(z_{1}, \cdots, z_{n}\right) \in \overline{\mathbb{K}}^{n}$. It follows that $\mathcal{F}[f]$ is well defined, continuous on $\overline{\mathbb{K}}^{n}$ and analytic on $\mathbb{K}^{n}$ by the Dominated convergence Theorem and Morera's Theorem.

For $n=1$ and $f$ locally integrable on $\mathbb{C}$ we see that

$$
\mathcal{F}[f](z)=\int_{0}^{\infty} \tilde{f}(r) r^{2 z+1} e^{-r^{2}} d r=M\left[\tilde{f} e^{-r^{2}}\right](2 z+2) \text { for } z \in \overline{\mathbb{K}}
$$


where $\tilde{f}(r)=\frac{1}{\pi} \int_{0}^{2 \pi} f\left(r e^{i \theta}\right) d \theta$, establishing a relationship between $\mathcal{F}(f)$ and the Mellin transform.

The next proposition is Proposition 3.1 of (Bauer and Le, 2011).

Proposition 2.5. Let $\mathcal{G}=\left\{\mathcal{F}[f]: f \in \mathcal{D}_{c}\right.$ for some $\left.c<1\right\}$ and suppose the function $G$ defined on $\overline{\mathbb{K}}^{n}$ is of the form $G=\sum_{i=1}^{m} u_{i} v_{i} p_{i}$, where $u_{i}, v_{i} \in \mathcal{G}$, for $i=1,2, \cdots, m$, and $p_{i}, i=1, \cdots$, m are polynomials. If $G \equiv 0$ on $\mathbb{N}^{n}$, then $G \equiv 0$ on $\overline{\mathbb{K}}^{n}$.

In one dimension, the following holds:

Corollary 2.6. Let $\psi, \phi \in S y m_{>0}\left(\mathbb{C}^{n}\right)$ be radial function, $k$ a positive integer and suppose

$$
\widetilde{\Phi(z)}:=M\left[\phi e^{-r^{2}}\right](2 z+k)\left(\frac{M\left(\psi e^{-r^{2}}\right)(2 z+k}{\Gamma(z+1)}-\frac{M\left[\psi e^{-r^{2}}\right](2 z+2 k)}{\Gamma(z+k)}\right)=0, \quad \operatorname{Re}(z)>-1,
$$

vanishes on the set $\{n+1, n+2, \cdots\}$. Then $\Phi(z)$ vanishes for all $z$ with $\operatorname{Re}(z)>0$.

Proof. The function $F(z):=\Gamma(z+k) \widetilde{\Phi(z)}$ vanishes on the set $\{n+1, n+2, \cdots\}$ and thus the function

$$
F(z+n)=\Gamma(z+n+k) M\left[\phi r^{2 n} e^{-r^{2}}\right](2 z+k)\left(\frac{M\left(\psi r^{2 n} e^{-r^{2}}\right)(2 z+k}{\Gamma(z+n+1)}-\frac{M\left[\psi r^{2 n} e^{-r^{2}}\right](2 z+2 k)}{\Gamma(z+n+k)}\right)
$$

vanishes in $\mathbb{N}$ and then by Proposition $2.5 F$ vanishes on $\overline{\mathbb{K}}$.

The following Lemma will be useful to us:

Lemma 2.7. Suppose $f$ and $g$ are holomorphic in the plane Rez $>0$ and satisfies $f(z+p) g(z)=f(z) g(z+p)$ for some $p \in \mathbb{N}$. Then $f(z+m p) g(z)=f(z) g(z+m p)$ for all $m \in \mathbb{N}$.

Proof. For $m \in \mathbb{N}$ we have that

$$
\prod_{l=1}^{m} f(z+l p) g(z+(l-1) p)=\prod_{l=1}^{m} f(z+(l-1) p) g(z+l p),
$$

and thus $f(z+m p) g(z)=f(z) g(z+m p)$, as required.

\section{Product of Toeplitz Operators with Radial Symbols}

In this section we will study the product problem, that is, when is the product of two Toeplitz operators a Toeplitz operator, and what is the resultant symbol.

We will frequently use the following lemma.

Lemma 3.1. Let $f \in \mathcal{D}_{c}, c<1$, be a radial function. Then for any $\alpha \in \mathbb{N}_{0}^{n}$

$$
\begin{aligned}
& T_{f}\left(z^{\alpha}\right)=\frac{1}{(n+|\alpha|-1) !} M\left[f e^{-r^{2}}\right](2(|\alpha|+n)) z^{\alpha} \\
& T_{f}\left(\bar{z}^{\alpha}\right)=\frac{1}{(n+|\alpha|-1) !} M\left[f e^{-r^{2}}\right](2(|\alpha|+n)) \bar{z}^{\alpha}
\end{aligned}
$$

Proof. Let $\alpha \in \mathbb{N}_{0}^{n}$. Then for any $\beta \in \mathbb{N}_{0}^{n}$ we have that

$$
\begin{aligned}
\left\langle T_{f} z^{\alpha}, z^{\beta}\right\rangle & =\left\langle f z^{\alpha}, z^{\beta}\right\rangle \\
& = \begin{cases}0 & \text { if } \alpha \neq \beta \\
\frac{2 n ! \alpha !}{(n+|\alpha|-1) !} M\left[f e^{-r^{2}}\right](2(|\alpha|+n)) & \text { if } \alpha=\beta\end{cases}
\end{aligned}
$$

and

which implies

$$
\left\langle z^{\alpha}, z^{\beta}\right\rangle= \begin{cases}0 & \text { if } \alpha \neq \beta \\ 2 n ! \alpha ! & \text { if } \alpha=\beta\end{cases}
$$

$$
\left\langle T_{f} z^{\alpha}, z^{\beta}\right\rangle=\frac{1}{(n+|\alpha|-1) !} M\left[f e^{-r^{2}}\right](2(|\alpha|+n))\left\langle z^{\alpha}, z^{\beta}\right\rangle .
$$


If $\beta \in \mathbb{N}^{n}$, then $\left\langle T_{f} z^{\alpha}, \bar{z}^{\beta}\right\rangle=0=\left\langle z^{\alpha}, \bar{z}^{\beta}\right\rangle$. This shows that

$$
\left\langle T_{f} z^{\alpha}, \bar{z}^{\beta}\right\rangle=\left\langle z^{\alpha}, \bar{z}^{\beta}\right\rangle, \quad \forall \beta \in \mathbb{N}^{n} .
$$

Since $\left\{\frac{1}{\sqrt{\alpha !}} z^{\alpha}\right\}_{\alpha \in \mathbb{N}_{0}^{n}} \cup\left\{\frac{1}{\sqrt{\alpha !}} \bar{z}^{\alpha}\right\}_{\alpha \in \mathbb{N}^{n}}$ is a basis for the pluriharmonic Fock space, we have

$$
T_{f}\left(z^{\alpha}\right)=\frac{1}{(n+|\alpha|-1) !} M\left[f e^{-r^{2}}\right](2(|\alpha|+n)) z^{\alpha} .
$$

By a similar argument we have that

$$
T_{f}\left(\bar{z}^{\alpha}\right)=\frac{1}{(n+|\alpha|-1) !} M\left[f e^{-r^{2}}\right](2(|\alpha|+n)) \bar{z}^{\alpha} .
$$

As an immediate consequence of Lemma 3.1 we have:

Proposition 3.2. Suppose $f, g \in \mathcal{S}$ are radial, then $T_{f} T_{g}=T_{g} T_{f}$ on the $\operatorname{span}\left\{\left\{e_{\alpha}\right\}_{\alpha \in \mathbb{N}_{0}^{n}}\right\}+\operatorname{span}\left\{\left\{\overline{e_{\alpha}}\right\}_{\alpha \in \mathbb{N} n}\right\}$, where

$$
\mathcal{S}:=\left\{g: \mathbb{C} \rightarrow \mathbb{C}: \exists C, c \text { such that }|g(z)| \leq C(1-|z|)^{c}\right\} .
$$

The next theorem is well known and the proof is similar to that in the Pluriharmonic Bergman space, see Theorem 5 of (Yang et al., 2013).

Theorem 3.3. Let $f \in \mathcal{D}_{c}, c<\frac{1}{2}$. Then the following are equivalent.

(1) For each multi-index $\alpha$, there exists $\lambda_{|\alpha|} \in \mathbb{C}$ depending on $|\alpha|$ such that $T_{f}\left(z^{\alpha}\right)=\lambda_{|\alpha|} z^{\alpha}, T_{f}\left(\bar{z}^{\alpha}\right)=\lambda_{|\alpha|} \bar{z}^{\alpha}$.

(2) $f$ is radial.

Corollary 3.4. Let $f, g \in S y m_{>0}\left(\mathbb{C}^{n}\right)$ be radial functions. If $T_{f} T_{g}=T_{h}$ then $h$ is radial.

Proof. By (11) we have

$$
\begin{aligned}
& T_{f} T_{g}\left(z^{\alpha}\right)=\frac{1}{((n+|\alpha|-1) !)^{2}} M\left[f e^{-r^{2}}\right](2(|\alpha|+n)) M\left[g e^{-r^{2}}\right](2(|\alpha|+n)) z^{\alpha} \\
& T_{f} T_{g}\left(\bar{z}^{\alpha}\right)=\frac{1}{((n+|\alpha|-1) !)^{2}} M\left[g e^{-r^{2}}\right](2(|\alpha|+n)) M\left[f e^{-r^{2}}\right](2(|\alpha|+n)) \bar{z}^{\alpha} .
\end{aligned}
$$

It follows from Theorem 3.3 that $h$ is radial.

Theorem 3.5. Let $f, g \in \mathcal{A}$ be radial functions. Then $T_{f} T_{g}$ is equal to the Toeplitz operator $T_{h}$ if and only if $h$ is the solution of the equation

$$
\left(e^{-r^{2}} f\right) *\left(e^{-r^{2}} g\right)=2\left(e^{-r^{2}}\right) *\left(h e^{-r^{2}}\right) .
$$

Proof Let $\alpha$ be any multi-index of non-negative integers. Then $T_{f} T_{g}=T_{h}$ implies

$$
T_{f} T_{g}\left(z^{\alpha}\right)=T_{h}\left(z^{\alpha}\right), \quad T_{f} T_{g}\left(\bar{z}^{\alpha}\right)=T_{h}\left(\bar{z}^{\alpha}\right) .
$$

It follows from (16) that

$$
\begin{aligned}
M\left[f e^{-r^{2}}\right](2(|\alpha|+n)) M\left[g e^{-r^{2}}\right](2(|\alpha|+n)) & =(n+|\alpha|-1) ! M\left[h e^{-r^{2}}\right](2(|\alpha|+n)) \\
& =2 M\left[e^{-r^{2}}\right](2(|\alpha|+n)) M\left[h e^{-r^{2}}\right](2(|\alpha|+n)) .
\end{aligned}
$$

Thus, the function

$$
\begin{aligned}
F(z) & =M\left[f e^{-r^{2}}\right](2 z) M\left[g e^{-r^{2}}\right](2 z)-M\left[2 e^{-r^{2}}\right](2 z) M\left[h e^{-r^{2}}\right](2 z) \\
& =M\left[\left(f e^{-r^{2}}\right) *\left(g e^{-r^{2}}\right)\right](2 z)-M\left[\left(2 e^{-r^{2}}\right) *\left(h e^{-r^{2}}\right)\right](2 z)
\end{aligned}
$$

is an entire function which vanishes on $\{k: k \in \mathbb{N}\}$. By Proposition 2.6 there exist functions $h_{1}, h_{2} \in \mathcal{A}$ such that $F(z)=M\left[\left(h_{1}-h_{2}\right) e^{-r}\right](2 z)$ and by Lemma 2.3 we have that $h_{1}=h_{2}$ on $\mathbb{R}^{+}$. This implies $\left(f e^{-r^{2}}\right) *\left(g e^{-r^{2}}\right)=2\left(e^{-r^{2}}\right) *\left(h e^{-r^{2}}\right)$, as required. 
Conversely, let $f, g \in \mathcal{A}$ and suppose there is an $h \in \mathcal{A}$ which satisfies (17). Then from (16) we have that

$$
\begin{aligned}
T_{f} T_{g}\left(z^{\alpha}\right) & =\frac{1}{((n+|\alpha|-1) !)^{2}} M\left[f e^{-r^{2}}\right](2(|\alpha|+n)) M\left[g e^{-r^{2}}\right](2(|\alpha|+n)) z^{\alpha} \\
& =\frac{1}{((n+|\alpha|-1) !)^{2}} M\left[\left(f e^{-r^{2}}\right) *\left(g e^{-r^{2}}\right)\right](2(|\alpha|+n)) z^{\alpha} \\
& =\frac{1}{((n+|\alpha|-1) !)^{2}} M\left[2\left(e^{-r^{2}}\right) *\left(h e^{-r^{2}}\right)\right](2(|\alpha|+n)) z^{\alpha} \\
& \left.=\frac{1}{((n+|\alpha|-1) !)^{2}} M\left[2 e^{-r^{2}}\right](2(|\alpha|+n)) M\left[h e^{-r^{2}}\right)\right](2(|\alpha|+n)) z^{\alpha} \\
& \left.=\frac{1}{(n+|\alpha|-1) !} M\left[h e^{-r^{2}}\right)\right](2(|\alpha|+n)) z^{\alpha}=T_{h}\left(z^{\alpha}\right), \alpha \in \mathbb{N}_{0}^{n} .
\end{aligned}
$$

Similarly, one can show that $T_{f} T_{g}\left(\bar{z}^{\alpha}\right)=T_{h}\left(\bar{z}^{\alpha}\right)$, for each $\alpha \in \mathbb{N}^{n}$. Thus $T_{f} T_{g}=T_{h}$.

\section{The Zero Product Problem}

We begin this section by observing that if $f \in \mathcal{D}_{c}, c<1$ then $T_{f}=0$ on $H_{p h}^{2}$ if and only if $f \equiv 0$ a.e on $\mathbb{C}^{n}$. Indeed, for any $h \in H^{2}$ we have that

$$
P(f h)(z)+\overline{P(\overline{f h})}(z)-P(f h)(0)=0, \text { for all } z \in \mathbb{C}^{n} .
$$

It follows that $\overline{P(\overline{f h})}(0)=0$ and thus $P(f h)(0)=0$. Thus (20) shows that $P(f h)(z)+\overline{P(\overline{f h})}(z)=0$ for all $z \in \mathbb{C}^{n}$ and $P(f h) \equiv 0$ for all $h \in H^{2}$ since $P(f h)(0)=0$. But this shows that the Toeplitz operator $Q_{f}: H^{2} \rightarrow H^{2}$ given by $Q_{f} g=P(f g)$ for $g \in H^{2}$ vanishes on $H^{2}$ and this implies that $f=0$ a.e on $\mathbb{C}^{n}$ by (Folland, 1989), page 140 .

The following is Lemma 3.2 of (Bauer and Le, 2011).

Lemma 4.1. Suppose $g \in L^{2}\left(\mathbb{C}^{n}, d \mu\right), l \in \mathbb{Z}^{n}, s \in \mathbb{Z}$ and let $(m-k) l:=\left(m_{1}-k_{1}\right) l_{1}+\cdots+\left(m_{n}-k_{n}\right) l_{n}$ for $m, k \in \mathbb{N}_{0}^{n}$. Then $\int_{\mathbb{C}^{n}} g(w) w^{m} \bar{w}^{k} d \mu(w)=0$ for all multi-indices $m, k \in \mathbb{N}_{0}^{n}$ such that $(m-k) l \neq s$ if and only if $g\left(\gamma^{l_{1}} z_{1}, \cdots, \gamma^{l_{n}} z_{n}\right)=\bar{\gamma}^{s} g(z)$ for a.e. $\gamma \in \mathbb{T}$ and a.e $z \in \mathbb{C}^{n}$.

For $g \in L^{2}\left(\mathbb{C}^{n}, d \mu\right)$ radial and $s \in \mathbb{C}$ such that $\operatorname{Re}(s)>-n$, we set $\omega(g, s)=M\left[2 g e^{-r^{2}}\right](2 s+2 n) / \Gamma(s+n)$. Then $\omega(g, s)$ is analytic on its domain and

$$
\omega(g,|\alpha|)=\frac{\mathcal{F}[g](\alpha)}{\alpha !}, T_{g} z^{\alpha}=\omega(g,|\alpha|) z^{\alpha} \text { and } T_{g} \bar{z}^{\alpha}=\omega(g,|\alpha|) \bar{z}^{\alpha},
$$

for every $\alpha \in \mathbb{N}_{0}^{n}$. Now, if $c \in \mathbb{C}$ then

$$
\begin{aligned}
\omega(g-c, s) & =M\left[2(g-c) e^{-r^{2}}\right](2 s+2 n) / \Gamma(s+n) \\
& =M\left[2 g e^{-r^{2}}\right](2 s+2 n) / \Gamma(s+n)-c M\left[2 e^{-r^{2}}\right](2 s+2 n) / \Gamma(s+n) \\
& =\omega(g, s)-c .
\end{aligned}
$$

It follows that $s \mapsto \omega(g, s)$ is constant if and only if $g$ is a constant function on $\mathbb{C}^{n}$.

Let $f, g \in S y m_{>0}\left(\mathbb{C}^{n}\right)$ be two radial functions, define the set

$$
\mathcal{Z}(f, g)=\{d \in \mathbb{Z}: \omega(f, s)=\omega(g, s+d) \text { for all } s \in \mathbb{K} \text { with } \operatorname{Re}(\mathrm{s}) \text { sufficiently large }\} .
$$

It is shown in (Bauer and Le, 2011), that if one of the functions is non-constant, then either $\mathcal{Z}(f, g)=\emptyset$ or $\mathcal{Z}(f, g)=\{d\}$ for some integer $d$. Also, if $f$ is non-constant then $\mathcal{Z}(f, f)=\{0\}$. Our next result was given in (Bauer and Le, 2011), in the case of the Fock space, and we show here that the result remains true on the Pluri-harmonic space.

Theorem 4.2. Suppose $f, g \in S y m_{>0}\left(\mathbb{C}^{n}\right)$ are radial functions, and at least one of them is non-constant. Then exactly one of the following assertions holds true:

(a) $\mathcal{Z}(f, g)=\emptyset$ and for any function $h \in S y m_{>0}\left(\mathbb{C}^{n}\right), T_{f} T_{h}=T_{h} T_{g}$ on $H_{p h}^{2}$ if and only if $h(z)=0$ a.e. $z \in \mathbb{C}^{n}$.

(b) $\mathcal{Z}(f, g)=\{d\}$ and for any function $h \in S y m_{>0}\left(\mathbb{C}^{n}\right), T_{f} T_{h}=T_{h} T_{g}$ on $H_{p h}^{2}$ if and only if $h(\gamma z)=\bar{\gamma}^{d} h(z)$ for a.e. $\gamma \in \mathbb{T}$ and a.e $z \in \mathbb{C}^{n}$. 
Proof. For any $\alpha, \beta \in \mathbb{N}^{n}$ we have the following:

$$
\begin{aligned}
& \text { (1) }\left\langle T_{f} T_{h} e_{\alpha}, e_{\beta}\right\rangle=\omega(f,|\beta|)\left\langle h e_{\alpha}, e_{\beta}\right\rangle \text { and }\left\langle T_{h} T_{g} e_{\alpha}, e_{\beta}\right\rangle=\omega(g,|\alpha|)\left\langle h e_{\alpha}, e_{\beta}\right\rangle ; \\
& \text { (2) }\left\langle T_{f} T_{h} e_{\alpha}, \bar{e}_{\beta}\right\rangle=\omega(f,|\beta|)\left\langle h e_{\alpha}, \bar{e}_{\beta}\right\rangle \text { and }\left\langle T_{h} T_{g} e_{\alpha}, \bar{e}_{\beta}\right\rangle=\omega(g,|\alpha|)\left\langle h e_{\alpha}, \bar{e}_{\beta}\right\rangle ; \\
& \text { (3) }\left\langle T_{f} T_{h} \bar{e}_{\alpha}, e_{\beta}\right\rangle=\omega(f,|\beta|)\left\langle h \bar{e}_{\alpha}, e_{\beta}\right\rangle \text { and }\left\langle T_{h} T_{g} \bar{e}_{\alpha}, e_{\beta}\right\rangle=\omega(g,|\alpha|)\left\langle h \bar{e}_{\alpha}, e_{\beta}\right\rangle ; \\
& \text { (4) }\left\langle T_{f} T_{h} \bar{e}_{\alpha}, \bar{e}_{\beta}\right\rangle=\omega(f,|\beta|)\left\langle h \bar{e}_{\alpha}, \bar{e}_{\beta}\right\rangle \text { and }\left\langle T_{h} T_{g} \bar{e}_{\alpha}, \bar{e}_{\beta}\right\rangle=\omega(g,|\alpha|)\left\langle h \bar{e}_{\alpha}, \bar{e}_{\beta}\right\rangle .
\end{aligned}
$$

Using (1)-(4) and the fact that $T_{f} T_{h}=T_{h} T_{g}$, we have the following:

$$
\begin{aligned}
& \left(1^{\prime}\right)(\omega(f,|\beta|)-\omega(g,|\alpha|))\left\langle h e_{\alpha}, e_{\beta}\right\rangle=0 ; \\
& \left(2^{\prime}\right)(\omega(f,|\beta|)-\omega(g,|\alpha|))\left\langle h e_{\alpha}, \bar{e}_{\beta}\right\rangle=0 ; \\
& \left(3^{\prime}\right)(\omega(f,|\beta|)-\omega(g,|\alpha|))\left\langle h \bar{e}_{\alpha}, e_{\beta}\right\rangle=0 ; \\
& \left(4^{\prime}\right)(\omega(f,|\beta|)-\omega(g,|\alpha|))\left\langle h \bar{e}_{\alpha}, \bar{e}_{\beta}\right\rangle=0 .
\end{aligned}
$$

Now, $\left(1^{\prime}\right)$ is equivalent to

$$
0=(\omega(f,|\beta|)-\omega(g,|\alpha|))\left\langle h e_{\alpha}, e_{\beta}\right\rangle=\frac{1}{\sqrt{\alpha ! \beta !}}(\omega(f,|\beta|)-\omega(g,|\alpha|)) \int_{\mathbb{C}^{n}} h(w) w^{\alpha} \bar{w}^{\beta} d \mu(w) .
$$

Let $m$ and $k$ be two fixed multi-indices in $\mathbb{N}_{0}^{n}$, with $\alpha=m+l$ and $\beta=k+l$ for $l \in \mathbb{N}_{0}^{n}$. Then by (21) we have

$$
\begin{aligned}
G_{1}(l) & :=\left(\frac{\mathcal{F}[f](k+l)}{(k+l) !}-\frac{\mathcal{F}[g](m+l)}{(m+l) !}\right) \int_{\mathbb{C}^{n}} h(w) w^{m} \bar{w}^{k}\left|w_{1}\right|^{2 l_{1}} \cdots\left|w_{n}\right|^{2 l_{n}} d \mu(w) \\
& =(\omega(f,|k|+|l|)-\omega(g,|m|+|l|)) \int_{\mathbb{C}^{n}} h(w) w^{m+l} \bar{w}^{k+l} d \mu(w)=0
\end{aligned}
$$

In a similar manner using $\left(2^{\prime}\right)-\left(4^{\prime}\right)$ we define $G_{i}(l), i=2,3,4$ for $l \in \mathbb{N}_{0}^{n}$. Now, it is easily seen that for each $i=1,2,3,4$, the function $(m+k+l) ! G_{i}(l)$ satisfies the hypothesis of Proposition 2.5. Therefore, $G_{i}(l)=0$ for all $l \in \mathbb{N}_{0}^{n}$ if and only if $G_{i}(l)=0$ for all $l \in \overline{\mathbb{K}}^{n}$. This by analyticity, is equivalent to either $\omega(f,|k|+|l|)=\omega(g,|m|+|l|)$ for all $l \in \overline{\mathbb{K}}^{n}$ or for $l \in \overline{\mathbb{K}}^{n}$ each of the following holds:

$$
\begin{aligned}
& \left(1^{\prime \prime}\right) \int_{\mathbb{C}^{n}} h(w) w^{m} \bar{w}^{k}\left|w^{l}\right|^{2} d \mu(w)=0 ; \\
& \left(2^{\prime \prime}\right) \int_{\mathbb{C}^{n}} h(w) w^{m+k+2 l} d \mu(w)=0 ; \\
& \left(3^{\prime \prime}\right) \int_{\mathbb{C}^{n}} h(w) \bar{w}^{m+k+2 l} d \mu(w)=0 ; \\
& \left(4^{\prime \prime}\right) \int_{\mathbb{C}^{n}} h(w) w^{k} \bar{w}^{m}\left|w^{l}\right|^{2} d \mu(w)=0 .
\end{aligned}
$$

Now, $\left(\omega(f,|k|+|l|)=\omega(g,|m|+|l|)\right.$ for all $l \in \overline{\mathbb{K}}^{n}$, implies that $|m|-|k| \in \mathcal{Z}(f, g)$. Since one of the functions $f, g$ is non-constant, we have the following two possibilities.

(a) If $\mathcal{Z}(f, g)=\emptyset$ then the conditions $\left(1^{\prime \prime}\right)-\left(4^{\prime \prime}\right)$ holds, and $\left(1^{\prime \prime}\right)$ is equivalent to $h(z)=0$ for a.e $z \in \mathbb{C}^{n}$.

The converse is immediate since $T_{f} T_{h}=0=T_{h} T_{g}$ whenever $h(z)=0$ for a.e $z \in \mathbb{C}^{n}$.

(b) If $\mathcal{Z}(f, g)=\{d\}$, then $\left(1^{\prime \prime}\right)-\left(4^{\prime \prime}\right)$ holds for all $l \in \overline{\mathbb{K}}^{n}$ satisfying $|m|-|k| \neq d$. By Lemma 4.1, assertions (1") and (4") is equivalent to $h(\gamma z)=\bar{\gamma}^{d} h(z)$ for a.e. $\gamma \in \mathbb{T}$ and a.e $z \in \mathbb{C}^{n}$. Conversely, suppose $h(\gamma z)=\bar{\gamma}^{d} h(z)$, for all $\gamma \in \mathbb{T}$. Then for every $m, k, l \in \mathbb{N}_{0}^{n}$

$$
\int_{\mathbb{C}^{n}} h(w) \bar{w}^{m+k+2 l} d \mu(w)=\bar{\gamma}^{d+|m+k+2 l|} \int_{\mathbb{C}^{n}} h(w) \bar{w}^{m+k+2 l} d \mu(w),
$$

and thus

$$
\left(1-\bar{\gamma}^{d+|m+k+2 l|}\right) \int_{\mathbb{C}^{n}} h(w) \bar{w}^{m+k+2 l} d \mu(w)=0
$$

for all $\gamma \in \mathbb{T}$. This gives ( $\left.3^{\prime \prime}\right)$. By observing that $h(\bar{\gamma} z)=\gamma^{d} h(z)$, for all $\gamma \in \mathbb{T}$, we similarly show that ( $\left.2^{\prime \prime}\right)$ holds for every $m, k, l \in \mathbb{N}_{0}^{n}$. Also Lemma 4.1, shows that ( $\left.1^{\prime \prime}\right)$ and (4") hold for any $m, k \in \mathbb{N}_{0}^{n}$ such that $|m|-|k| \neq d$. This shows that for 
all $m, k, k_{1}, k_{2} \in \mathbb{N}_{0}^{n}$, we have

$$
\begin{aligned}
& \left(1^{\prime \prime \prime}\right)\left((\omega(f,|k|+|l|)-\omega(g,|m|+|l|)) \int_{\mathbb{C}^{n}} h(w) w^{m} \bar{w}^{k}\left|w^{l}\right|^{2} d \mu(w)=0 ;\right. \\
& \left(2^{\prime \prime \prime}\right)\left(\left(\omega\left(f,\left|k_{1}\right|+|l|\right)-\omega(g,|m|+|l|)\right) \int_{\mathbb{C}^{n}} h(w) w^{m+k_{1}+2 l} d \mu(w)=0 ;\right. \\
& \left(3^{\prime \prime \prime}\right)\left(\left(\omega\left(f,\left|k_{2}\right|+|l|\right)-\omega(g,|m|+|l|)\right) \int_{\mathbb{C}^{n}} h(w) \bar{w}^{m+k_{2}+2 l} d \mu(w)=0 ;\right. \\
& \left(4^{\prime \prime \prime}\right)\left((\omega(f,|k|+|l|)-\omega(g,|m|+|l|)) \int_{\mathbb{C}^{n}} h(w) w^{k} \bar{w}^{m}\left|w^{l}\right|^{2} d \mu(w)=0 .\right.
\end{aligned}
$$

for every $l \in \mathbb{N}_{0}^{n}$. Now $\left(1^{\prime \prime \prime}\right)-\left(4^{\prime \prime \prime}\right)$ implies that, for every $\alpha, \beta, \gamma, \lambda \in \mathbb{N}_{0}^{n}$ we have

$$
\left\langle T_{f} T_{h} e_{\alpha}-T_{h} T_{g} e_{\alpha}, e_{\beta}\right\rangle=0=\left\langle T_{f} T_{h} e_{\alpha}-T_{h} T_{g} e_{\alpha}, \bar{e}_{\gamma}\right\rangle,
$$

and

$$
\left\langle T_{f} T_{h} \bar{e}_{\alpha}-T_{h} T_{g} \bar{e}_{\alpha}, e_{\lambda}\right\rangle=0=\left\langle T_{f} T_{h} \bar{e}_{\alpha}-T_{h} T_{g} \bar{e}_{\alpha}, \bar{e}_{\beta}\right\rangle
$$

This shows that

$$
\left\langle T_{f} T_{h} e_{\alpha}-T_{h} T_{g} e_{\alpha}, e_{\beta}+\bar{e}_{\gamma}\right\rangle=0 \text { and }\left\langle T_{f} T_{h} \bar{e}_{\alpha}-T_{h} T_{g} \bar{e}_{\alpha}, e_{\lambda}+\bar{e}_{\beta}\right\rangle=0
$$

for every $\alpha, \beta, \gamma, \lambda \in \mathbb{N}_{0}^{n}$. It follows that $T_{f} T_{h} e_{\alpha}=T_{h} T_{g} e_{\alpha}$ and $T_{f} T_{h} \bar{e}_{\alpha}=T_{h} T_{g} \bar{e}_{\alpha}$ for every $\alpha \in \mathbb{N}$. We therefore conclude that $T_{f} T_{h}=T_{h} T_{g}$, as required.

Corollary 4.3. Suppose $f, g \in S y m_{>0}\left(\mathbb{C}^{n}\right)$ with $f$ radial. If $T_{f} T_{g}=0$ or $T_{g} T_{f}=0$ on $H_{p h}^{2}$ then either $f$ or $g$ must be zero a.e on $\mathbb{C}^{n}$.

Proof If $f$ is a constant, then $T_{f} T_{g}$ and $T_{g} T_{f}$ are constant multiples of $T_{g}$. Now $T_{g}=0$, implies $g(z)=0$ for a.e $z \in \mathbb{C}^{n}$, by remark in the begining of this section. Now suppose $f$ is a non-constant function. Take $h(z)=0$ for all $z \in \mathbb{C}^{n}$. If $T_{f} T_{g}=0$ then $T_{f} T_{g}=T_{g} T_{h}$ and since $\mathcal{Z}(f, h)=\emptyset$ we see by Theorem 4.2 that $g$ must be zero. The case $T_{g} T_{f}=0$ is similar.

\section{Commuting Toeplitz Operators}

We will study the commutators of two Toeplitz operators with quasi homogeneous symbols. Our next theorem is a corollary of Theorem 4.2.

Theorem 5.1. Let $f \in S y m_{>0}\left(\mathbb{C}^{n}\right)$ be a radial and non-constant function. Then for any $g \in S y m_{>0}\left(\mathbb{C}^{n}\right), T_{f}$ and $T_{g}$ commute if and only if $g(\gamma z)=g(z)$, for a.e. $\gamma \in \mathbb{T}$ and a.e $z \in \mathbb{C}^{n}$.

Proof Since $\mathcal{Z}(f, f)=\{0\}$, Theorem 4.2 implies that $T_{f} T_{g}=T_{g} T_{f}$ if and only if $g(\gamma z)=g(z)$, for a.e. $\gamma \in \mathbb{T}$ and a.e $z \in \mathbb{C}^{n}$.

Recall that Theorem 5.1 also holds for the setting of the Fock space, see (Bauer and Lee, 2011), for the details. Based on the above theorem, we have the following corollary.

Corollary 5.2. Let $p, s \in \mathbb{N}^{n}$ and suppose $\psi, \phi \in S y m_{>0}\left(\mathbb{C}^{n}\right)$ are radial functions. Then $T_{\psi} T_{\xi^{p} \bar{\xi}^{s} \phi}=T_{\xi^{p} \bar{\xi}^{s} \phi} T_{\psi}$ on $H_{p h}^{2}$ if and only if either $|p|=|s|$ or $\phi=0$.

Proof. Let $g(z)=\xi^{p} \bar{\xi}^{s} \phi(|z|)$. Then for $\gamma \in \mathbb{T}, g(\gamma z)=\gamma^{|p|-|s|} \xi^{p} \bar{\xi}^{s} \phi(|z|)$ and thus $g(\gamma z)=g(z)$ if and only if either $\phi \equiv 0$ or $|p|=|s|$. The result follows from Theorem 5.1.

With Theorem 5.1 one easily shows that the result on commutativity of Toeplitz operators given in (Bauer and Lee, 2011), for the Fock space also holds for the Pluriharmonic Fock space. We present here the result for completeness. Let $n=1$ and define $\mathcal{D}_{c}$ on $\mathbb{C}$. The following is Lemma 2 of (Bauer and Issa, 2012).

Lemma 5.3. Let $c<\frac{1}{2}$ and $f \in \mathcal{D}_{c}$. Then $f$ has the series expansion:

$$
f\left(r e^{i \theta}\right)=\sum_{k=-\infty}^{\infty} f_{k}(r) e^{i k \theta}
$$

with $\left|f_{k}(r)\right| \leq C e^{c r^{2}}$ for some constant $C>0$ and for almost all $r \in(0, \infty)$. The above convergence holds in $L^{2}\left(\mathbb{C}, d \mu_{\delta}\right)$ for $\delta>0$. 
We have the following:

Corollary 5.4. Suppose $u \in S y m_{>0}(\mathbb{C})$ is radial and let $v \in S y m_{>0}(\mathbb{C})$ have the expansion

$$
v\left(r e^{i \theta}\right)=\sum_{j=-\infty}^{\infty} v_{j}(r) e^{i j \theta}
$$

Then the following are equivalent:

(a) $T_{u} T_{v}=T_{v} T_{u}$;

(b) $T_{u} T_{v_{j}} e^{i j \theta}=T_{v_{j}} e^{i j \theta} T_{u}$ for every $j \in \mathbb{Z}$;

(c) $v_{j}=0$ for $j \in \mathbb{Z} \backslash\{0\}$;

(d) $v$ is radial.

Proof. By Theorem 5.1 (a) holds if and only if $v\left(r e^{i(\theta+\phi)}\right)=v\left(r e^{i \theta}\right)$ for every $\phi \in[0,2 \pi]$ if and only if $\left(e^{j i \phi}-1\right) v_{j}=0$ for all $j \in \mathbb{Z}$ if and only if $v_{j}=0$ for all $j \in \mathbb{Z} \backslash\{0\}$. But $v_{j}=0$ for all $j \in \mathbb{Z} \backslash\{0\}$ is equivalent to $v$ is radial and also equivalent to (b).

We remark that Corollary 5.2 was first given in (Guan et al., 2013), in the case of the Pluri-harmonic Bergman space, with the requirement that $p \perp s$. In our case that restriction is not required. In addition, the corollary also holds in the case of the Fock space. We also note that Corollary 5.4 has been given in (Bauer and Lee, 2011), in the case of the Fock space. However in their setting $u$ and $v$ are from the class $\mathcal{S}$ given by (15). However, the above corollary shows that the result also hold for the Fock space with $u, v \in S y m_{>0}(\mathbb{C})$.

To end this section we will tend our attention to the case when both symbols are quasihomogeneous of degree ( $p, s)$. We begin with the following Lemma which will be useful to us throughout the remaining of this section.

Lemma 5.5. Let $p, s$ be two multi-indexes of non-negative integers and $\phi$ is a radial function in $\mathcal{D}_{c}$, $c<\frac{1}{2}$. Then for any multi-index $\alpha$,

$$
\begin{gathered}
T_{\xi^{p} \bar{\xi}^{s} \phi}\left(z^{\alpha}\right)= \begin{cases}\frac{2(p+\alpha) ! M\left[\phi e^{-r^{2}}\right](2|\alpha|+|p|-|s|+2 n)}{(n+|p+\alpha|-1) !(p+\alpha-s) !} z^{\alpha+p-s}, & \text { if } p+\alpha \gtrsim s \\
\frac{2 s ! M\left[\phi e^{-r^{2}}\right](|s|-|p|+2 n)}{(s-p-\alpha) !(n+|s|-1) !} \bar{z}^{s-p-\alpha}, & \text { if } p+\alpha \lesssim s \\
0, & \text { otherwise. }\end{cases} \\
T_{\xi^{p} \bar{\xi}^{s} \phi}\left(\bar{z}^{\alpha}\right)= \begin{cases}\frac{2(s+\alpha) ! M\left[\phi e^{-r^{2}}\right](2|\alpha|+|s|-|p|+2 n)}{(n+|s+\alpha|-1) !(s+\alpha-p) !} \bar{z}^{\alpha+p-s}, & \text { if } s+\alpha \gtrsim p \\
\frac{2 p ! M\left[\phi e^{-r^{2}}\right](|p|-|s|+2 n)}{(p-s-\alpha) !(n+|p|-1) !} \bar{z}^{p-s-\alpha}, & \text { if } s+\alpha \lesssim p \\
0, & \text { otherwise. }\end{cases}
\end{gathered}
$$

In particular, if $p$, s are two non-zero multi-indexes with $p \perp s$, the we have

$$
\begin{aligned}
& T_{\xi^{p} \bar{\xi}^{s} \phi}\left(z^{\alpha}\right)= \begin{cases}\frac{2(p+\alpha) ! M\left[\phi e^{-r^{2}}\right](2|\alpha|+|p|-|s|+2 n)}{(n+|p+\alpha|-1) !(p+\alpha-s) !} z^{\alpha+p-s,}, & \text { if } \alpha \gtrsim s \\
0, & \text { otherwise. }\end{cases} \\
& T_{\xi^{p} \bar{\xi}^{s} \phi}\left(\bar{z}^{\alpha}\right)= \begin{cases}\frac{2(s+\alpha) ! M\left[\phi e^{-r^{2}}\right](2|\alpha|+|s|-|p|+2 n)}{(n+|s+\alpha|-1) !(s+\alpha-p) !} \bar{z}^{\alpha+p-s}, & \text { if } \alpha \gtrsim p \\
0, & \text { otherwise. }\end{cases}
\end{aligned}
$$

Proof. If $\phi$ is a radial function in $\mathcal{D}_{c}, c<\frac{1}{2}$ then for each $\beta \in \mathbb{N}^{n}$ we have that

$$
\left\langle P\left(\xi^{p} \bar{\xi}^{s} \phi z^{\alpha}\right), z^{\beta}\right\rangle= \begin{cases}\frac{2 n !(p+\alpha) ! M\left[\phi e^{-r^{2}}\right](2|\alpha|+|p|-|s|+2 n)}{(n+|p+\alpha|-1) !(s+\alpha-p) !}, & \text { if } \beta=p+\alpha-s \\ 0, & \text { otherwise. }\end{cases}
$$

If $p+\alpha-s$ is a multi-index of non-negative integers, then for each $\beta \in \mathbb{N}_{0}^{n}$ we have that

$$
\left\langle z^{p+\alpha-s}, z^{\beta}\right\rangle= \begin{cases}n !(p+\alpha-s) !, & \text { if } \beta=p+\alpha-s \\ 0, & \text { otherwise }\end{cases}
$$


Also for $|\beta| \neq 0$, then $\left\langle P\left(\xi^{p} \bar{\xi}^{s} \phi z^{\alpha}\right), \bar{z}^{\beta}\right\rangle=0=\left\langle z^{p+\alpha-s}, \bar{z}^{\beta}\right\rangle$. Thus,

$$
P\left(\xi^{p} \bar{\xi}^{s} \phi z^{\alpha}\right)=\left\{\begin{array}{lc}
\frac{2(p+\alpha) ! M\left[\phi e^{-r^{2}}\right](2|\alpha|+|p|-|s|+2 n)}{(n+|p+\alpha|-1) !(p+\alpha-s) !} z^{\alpha+p-s}, & \text { if } p+\alpha \gtrsim s \\
0, & \text { otherwise }
\end{array}\right.
$$

Using the same arguments as above, we have that

$$
P\left(\xi^{s} \bar{\xi}^{p} \bar{\phi}_{\bar{z}}^{\alpha}\right)= \begin{cases}\frac{2 s ! M\left[\phi e^{-r^{2}}\right](2|| s|-| p \mid+2 n)}{(n+|s|-1) !(s-\alpha-p) !} z^{\alpha+p-s}, & \text { if } p+\alpha \lesssim s \\ 0, & \text { otherwise }\end{cases}
$$

Thus, for $\alpha \in \mathbb{N}_{0}^{n}$ we have

$$
\begin{aligned}
T_{\xi p} \bar{\xi}^{s} z^{\alpha} & =P\left(\xi^{p} \bar{\xi}^{s} \phi z^{\alpha}\right)+\overline{P\left(\xi s \bar{\xi}^{p} \bar{\phi} \bar{z}^{\alpha}\right)}-P\left(\xi^{p} \bar{\xi}^{s} \phi z^{\alpha}\right)(0) \\
& = \begin{cases}\frac{2(p+\alpha) ! M\left[\phi e^{-r^{2}}\right](2|\alpha|+|p|-|s|+2 n)}{(n+|p+\alpha|-1) !(p+\alpha-s) !} z^{\alpha+p-s}, & \text { if } p+\alpha \gtrsim s \\
\frac{2 s ! M\left[\phi e^{-r^{2}}\right](2|| s|-| p \mid+2 n)}{(n+|s|-1) !(s-\alpha-p) !} z^{\alpha+p-s}, & \text { if } p+\alpha \lesssim s \\
0, & \text { otherwise, }\end{cases}
\end{aligned}
$$

which is (42). If $p \perp s$ then $p+\alpha \gtrsim s$ is equivalent to $\alpha \gtrsim s$. Indeed, since $p, s$ are non-zero with $p \perp s$, there exists $i \in\{1, \cdots, n\}$ such that $p_{i} \neq 0$ and $s_{i}=0$, that is there does not exist multi-index $\alpha$ such that $p+\alpha \lesssim s$. This shows that

$$
T_{\xi^{p} \bar{\xi}^{s} \phi} z^{\alpha}= \begin{cases}\frac{2(p+\alpha) ! M\left[\phi e^{-r^{2}}\right](2|\alpha|+|p|-|s|+2 n)}{(n+|p+\alpha|-1) !(p+\alpha-s) !} z^{\alpha+p-s}, & \text { if } \alpha \gtrsim s \\ 0, & \text { otherwise }\end{cases}
$$

which is (27). In a similar, one can prove (26) and (28) and so we omit the details here.

Theorem 5.6. Let $p, s \in \mathbb{N}^{n}$ be such that $p \perp s$ and $\psi$ and $\phi$ be two radial functions in $\mathcal{A}$. If $0<\|p|-| s\| \leq 2$ then $T_{\xi^{p} \bar{\xi}^{s} \psi} T_{\xi^{p} \bar{\xi}^{s} \phi}=T_{\xi^{p} \bar{\xi}_{\phi}^{s}} T_{\xi^{p} \bar{\xi}^{s} \psi}$ if and only if $\phi=C \psi$ for some constant $C$.

Proof. Direct calculation using Lemma 5.5 gives

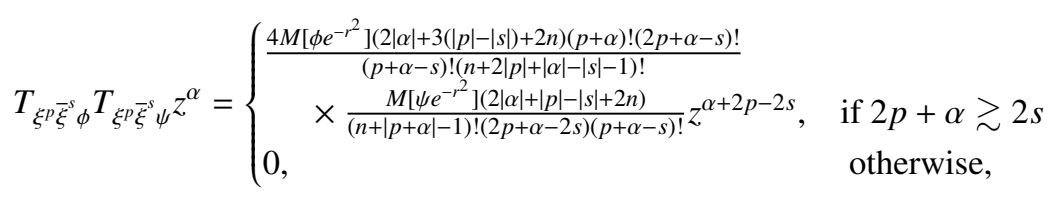

$$
\begin{aligned}
& T_{\xi^{p} \bar{\xi}^{s} \phi} T_{\xi^{p} \bar{\xi}^{s} \psi^{\alpha}} \bar{z}^{\alpha}= \begin{cases}\frac{4 M\left[\phi e^{-r^{2}}\right](2|\alpha|+3(|s|-|p|)+2 n)(s+\alpha) !(2 p+\alpha-s) !}{(s+\alpha-p) !(n+2|s|+|\alpha|-|p|-1) !} & \\
\quad \times \frac{M\left[\psi e^{-r^{2}}\right](2|\alpha|+|s|-|p|+2 n)}{(n+|s+\alpha|-1) !(2 s+\alpha-2 p)} \bar{z}^{\alpha+2 s-2 p}, & \text { if } 2 s+\alpha \gtrsim 2 p \\
0, & \text { otherwise, }\end{cases}
\end{aligned}
$$

and

$$
\begin{aligned}
& T_{\xi^{p} \bar{\xi}^{s} \psi} T_{\xi^{p} \bar{\xi}^{s} \phi^{\alpha}} z^{\alpha}= \begin{cases}\frac{4 M\left[\psi e^{-r^{2}}\right](2|\alpha|+3(|p|-|s|)+2 n)(p+\alpha) !(2 p+\alpha-s) !}{(p+\alpha-s) !(p+\alpha-s) !(n+2|p|+|\alpha|-|s|-1) !} & \\
\quad \times \frac{M\left[\phi e^{-r^{2}}\right](2|\alpha|+|p|-|s|+2 n)}{(n+|p+\alpha|-1) !(2 p+\alpha-2 s) !} z^{\alpha+2 p-2 s}, & \text { if } 2 p+\alpha \gtrsim 2 s \\
0, & \text { otherwise, }\end{cases} \\
& T_{\xi^{p} \bar{\xi}^{s} \psi} T_{\xi^{p} \bar{\xi}^{s} \phi} \bar{z}^{\alpha}= \begin{cases}\frac{4 M\left[\psi e^{-r^{2}}\right](2|\alpha|+3(|s|-|p|)+2 n)(s+\alpha) !(2 p+\alpha-s) !}{(s+\alpha-p) !(n+2|s|+|\alpha|-|p|-1) !} & \\
\quad \times \frac{M\left[\phi e^{-r^{2}}\right](2|\alpha|+|s|-|p|+2 n)}{(n+|s+\alpha|-1) !(2 s+\alpha-2 p) !} \bar{z}^{\alpha+2 s-2 p}, & \text { if } 2 s+\alpha \gtrsim 2 p \\
0, & \text { otherwise. }\end{cases}
\end{aligned}
$$

Thus, if $T_{\xi^{p} \bar{\xi}^{s} \phi} T_{\xi^{p} \bar{\xi}^{s} \psi}=T_{\xi^{p} \bar{\xi}^{s} \psi} T_{\xi^{p} \bar{\xi}^{s} \phi}$, then we have

$$
\begin{aligned}
& M\left[\psi e^{-r^{2}}\right](2|\alpha|+|s|-|p|+2 n) M\left[\phi e^{-r^{2}}\right](2|\alpha|+3(|s|-|p|)+2 n)= \\
& M\left[\phi e^{-r^{2}}\right](2|\alpha|+|s|-|p|+2 n) M\left[\psi e^{-r^{2}}\right](2|\alpha|+3(|s|-|p|)+2 n) \text { if } 2 s+\alpha \gtrsim 2 p
\end{aligned}
$$


and

$$
\begin{aligned}
& M\left[\psi e^{-r^{2}}\right](2|\alpha|+|p|-|s|+2 n) M\left[\phi e^{-r^{2}}\right](2|\alpha|+3(|p|-|s|)+2 n)= \\
& M\left[\phi e^{-r^{2}}\right](2|\alpha|+|p|-|s|+2 n) M\left[\psi e^{-r^{2}}\right](2|\alpha|+3(|p|-|s|)+2 n) \text { if } 2 p+\alpha \gtrsim 2 s .
\end{aligned}
$$

Now assume $|s|-|p|=k \in\{1,2\}$, then (36) implies

$$
\begin{aligned}
& M\left[\psi e^{-r^{2}} r^{2 n+k}\right](2|\alpha|) M\left[\phi e^{-r^{2}} r^{2 n+k}\right](2(|\alpha|+k))= \\
& M\left[\phi e^{-r^{2}} r^{2 n+k}\right](2|\alpha|) M\left[\psi e^{-r^{2}} r^{2 n+k}\right](2(|\alpha|+k)), \text { if } \alpha \gtrsim 2 p .
\end{aligned}
$$

Thus the function

$$
F(z)=M\left[\psi e^{-r^{2}} r^{2 n+k}\right](2 z) M\left[\phi e^{-r^{2}} r^{2 n+3 k}\right](2 z)-M\left[\phi e^{-r^{2}} r^{2 n+k}\right](2 z) M\left[\psi e^{-r^{2}} r^{2 n+3 k}\right](2 z),
$$

is holomomorphic for all $z$ with $\operatorname{Re} z>0$ and vanishes on $m=\{2|p|, 2|p|+1, \cdots\}$. By Proposition 2.2, there exist $h_{1}, h_{2} \in \mathcal{A}$ such that $F(z)=M\left[\left(h_{1}-h_{2}\right) e^{-r}\right](2 z)=M\left[h e^{-r}\right](2 z)$. Since $M\left[h e^{-r}\right](2 m)=0$ for all $m \in \mathbb{N}$, Lemma 2.3 shows that $h=0$ a.e on $\mathbb{R}^{+}$and thus $F(z)=0$ for all $z$ with $\operatorname{Re} z>0$. Thus

$$
M\left[\psi e^{-r^{2}} r^{2 n+k}\right](2 z) M\left[\phi e^{-r^{2}} r^{2 n+k}\right](2(z+k))=M\left[\phi e^{-r^{2}} r^{2 n+k}\right](2 z) M\left[\psi e^{-r^{2}} r^{2 n+k}\right](2(z+k)) .
$$

That is

$$
M\left[\widetilde{\psi} e^{-r} r^{n+\frac{k}{2}}\right](z) M\left[\widetilde{\phi} e^{-r} r^{n+\frac{k}{2}}\right](z+k)=M\left[\widetilde{\phi} e^{-r} r^{n+\frac{k}{2}}\right](z) M\left[\widetilde{\psi} e^{-r} r^{n+\frac{k}{2}}\right](z+k),
$$

where $\widetilde{\psi}(r)=\psi(\sqrt{r})$. By Lemma 2.7 we have that

$$
M\left[\widetilde{\psi} e^{-r} r^{n+\frac{k}{2}}\right](z) M\left[\widetilde{\phi} e^{-r} r^{n+\frac{k}{2}}\right](z+m k)=M\left[\widetilde{\phi} e^{-r} r^{n+\frac{k}{2}}\right](z) M\left[\widetilde{\psi} e^{-r} r^{n+\frac{k}{2}}\right](z+m k),
$$

for all $m \in \mathbb{N}$. Choose $z_{0} \in \mathbb{C}$ with $\operatorname{Re} z_{0}>0$ such that $M\left[\widetilde{\psi} e^{-r} r^{n+\frac{k}{2}}\right]\left(z_{0}\right) \neq 0$. Then (40) implies that

$$
M\left[\widetilde{\phi} e^{-r} r^{n+z_{0}-1+\frac{k}{2}}\right](1+m k)=C M\left[\widetilde{\psi} e^{-r} r^{n+z_{0}-1+\frac{k}{2}}\right](1+m k),
$$

for all $m \in \mathbb{N}$, where $C=C\left(z_{0}, \widetilde{\phi}, \widetilde{\psi}\right)=\frac{M\left[\widetilde{\phi} e^{-r} r^{n+\frac{k}{2}}\right]\left(z_{0}\right)}{M\left[\widetilde{\psi} e^{-r} r^{n+\frac{k}{2}}\right]\left(z_{0}\right)}$. Thus $M\left[(\widetilde{\phi}-C \widetilde{\psi}) e^{-r} r^{n+z_{0}-1+\frac{k}{2}}\right](1+m k)=0$ for $m=1,2, \cdots$. Since $k \leq 2$, Lemma 2.3 shows that $\widetilde{\phi}=C \psi$, as required. In a similar manner, we show that the result holds in the case $|p|-|s|>0$ by using equation (37) intead of (36).

The proof of the converse is trivial.

Corollary 5.7. Let $p, s \in \mathbb{N}^{n}$ be such that $p \perp s$ and $\psi$ is radial and belongs to $\mathcal{A}$. Suppose $\phi \in \mathcal{A}$ is radial. If $0<\|p|-| s\| \leq 2$ then $T_{\xi^{p} \bar{\xi}^{s} \psi} T_{\xi^{p} \bar{\xi}^{s} \phi}=T_{\xi^{p} \bar{\xi}^{s} \phi} T_{\xi^{p} \bar{\xi}^{s} \psi}$ on the Fock space $H^{2}$ if and only if $\phi=C \psi$ for some constant $C$.

\section{Finite Rank Toeplitz Operators}

We give here some results on the finite rank of Toeplitz operators with quasihomogeneous symbols and on the commutator operator. Recall that if $f, g \in \mathrm{Sym}_{>0}\left(\mathbb{C}^{n}\right)$ then the commutator $\left[T_{f}, T_{g}\right]$ is defined by

$$
\left[T_{f}, T_{g}\right]=T_{f} T_{g}-T_{g} T_{f} .
$$

Theorem 6.1. Let $p_{1}, p_{2}, s_{1}, s_{2}$ be multi-indices and let $\phi_{1}, \phi_{2}$ be radial functions in $S_{y m}{ }_{>0}\left(\mathbb{C}^{n}\right)$. Then $T_{\xi^{p_{2}} \bar{\xi}^{s_{2}} \phi_{2}} T_{\xi^{p} 1 \bar{\xi}^{s_{1}} \phi_{1}}$ is of finite rank if either $\phi_{1} \equiv 0$ or $\phi_{2} \equiv 0$.

Proof. Let $S=T_{\xi^{p} \bar{\xi}^{s_{2}} \phi_{2}} T_{\xi^{p} 1 \bar{\xi}^{s_{1}} \phi_{1}}$. Then by Lemma 5.5 we have that

$$
S\left(z^{\alpha}\right)= \begin{cases}\frac{4\left(p_{1}+\alpha\right) !\left(p_{2}+\alpha+p_{1}-s_{1}\right) ! M\left[\phi_{1} e^{-r^{2}}\right]\left(2|\alpha|+\left|p_{1}\right|-\left|s_{1}\right|+2 n\right)}{\left(n+\left|p_{1}+\alpha\right|-1\right) !\left(n+\left|p_{2}+\alpha+p_{1}-s_{1}\right|-1\right) !\left(p_{1}+\alpha-s_{1}\right) !} & \\ \frac{M\left[\phi_{2} e^{-r^{2}}\right]\left(2|\alpha|+\left|p_{1}\right|-\left|s_{1}\right|+\left|p_{2}\right|-\left|s_{2}\right|+2 n\right)}{\left(p_{2}+\alpha+p_{1}+-s_{2}-s_{1}\right) !} z^{\alpha+p_{1}+p_{2}-s_{1}-s_{2}}, & \text { if } p_{1}+\alpha \gtrsim s_{1}, p_{1}+p_{2}+\alpha \gtrsim s_{1}+s_{2} \\ \frac{4\left(s_{2}+s_{1}+\alpha-p_{1}\right) ! s ! M\left[\phi_{1} e^{-r^{2}}\right]\left(\left|s_{1}\right|-\left|p_{1}\right|+2 n\right)}{\left(s_{1}-p_{1}-\alpha\right) !\left(n+s_{1} \mid-1\right) !\left(s_{2}+s_{1}+\alpha-p_{1}-p_{2}\right) !} & \\ \frac{M\left[\phi_{2} e^{-r^{2}}\right]\left(2\left|s_{1}-p_{1}+\alpha\right|+\left|s_{2}\right|-\left|p_{2}\right|+2 n\right)}{\left(n+\left|s_{2}+s_{1}-p_{1}+\alpha\right|-1\right) !} \bar{z}^{s_{1}-p_{1}-\alpha+p_{2}-s_{2}}, & \text { if } p_{1}+\alpha \lesssim s_{1}, p_{1}+p_{2}-\alpha \lesssim s_{1}+s_{2} \\ 0, & \text { otherwise. }\end{cases}
$$


and

$$
S\left(\bar{z}^{\alpha}\right)= \begin{cases}\frac{4\left(s_{1}+\alpha\right) !\left(s_{2}+\alpha+s_{1}-p_{1}\right) ! M\left[\phi_{1} e^{-r^{2}}\right]\left(2|\alpha|+\left|s_{1}\right|-\left|p_{1}\right|+2 n\right)}{\left(n+\left|s_{1}+\alpha\right|-1\right) !\left(n+\left|s_{2}+\alpha-p_{1}\right|-1\right) !\left(s_{1}+\alpha-p_{1}\right) !} & \\ \frac{M\left[\phi_{2} e^{-r^{2}}\right]\left(2\left|\alpha+s_{1}-p_{1}\right|+\left|s_{2}\right|-\left|p_{2}\right|+2 n\right)}{\left(s_{2}+\alpha+s_{1}-p_{1}-p_{2}\right) !} \bar{z}^{\alpha+s_{1}-p_{1}+s_{2}-p_{2}}, & \text { if } s_{1}+\alpha \gtrsim p_{1}, \alpha+s_{1}+s_{2} \gtrsim p_{1}+p_{2} \\ \frac{4 p_{1} !\left(p_{2}+p_{1}-s_{1}-\alpha\right) ! M\left[\phi_{1} e^{-r^{2}}\right]\left(\left|p_{1}\right|-\left|s_{1}\right|+2 n\right)}{\left(p_{1}-s_{1}-\alpha\right) !\left(n-\left|p_{2}+p_{1}-s_{1}-\alpha\right|-1\right) !\left(n+\left|p_{1}\right|-1\right) !} & \\ \frac{M\left[\phi_{2} e^{-r^{2}}\right]\left(\left|p_{1}-s_{1}-\alpha\right|-\left|s_{2}\right|+2 n\right)}{\left(p_{2}+p_{1}-s_{1}-s_{2}-\alpha\right) !} z^{p_{1}-s_{1}-\alpha+p_{2}-s_{2}}, & \text { if } s_{1}+\alpha \lesssim p_{1}, s_{1}+s_{2}+\alpha \lesssim p_{1}+p_{2} \\ 0, & \text { otherwise. }\end{cases}
$$

Since $S$ has finite rank, we have that $\left\{S\left(z^{\alpha}\right)\right\}_{\alpha \in \mathbb{N}^{n}}$ and $\left\{S\left(\bar{z}^{\alpha}\right)\right\}_{\alpha \in \mathbb{N}^{n}}$ must have finite rank. It follows that there exists $\alpha_{0} \in \mathbb{N}^{n}$ such that $p_{1}+\alpha_{0} \gtrsim s_{1}, p_{2}+p_{1}+\alpha_{0} \gtrsim s_{1}+s_{2}$ and $S\left(z^{\alpha}\right)=0$ for all $\alpha \gtrsim \alpha_{0}$. That is

$$
M\left[\phi_{1} e^{-r^{2}}\right]\left(2|\alpha|+\left|p_{1}\right|-\left|s_{1}\right|+2 n\right) M\left[\phi_{2} e^{-r^{2}}\right]\left(2|\alpha|+\left|p_{1}\right|-\left|s_{1}\right|+\left|p_{2}\right|-\left|s_{2}\right|+2 n\right)=0
$$

for all $\alpha \gtrsim \alpha_{0}$. Let $k=\left|p_{1}\right|-\left|s_{1}\right|$ and $m=\left|p_{1}\right|-\left|s_{1}\right|+\left|p_{2}\right|-\left|s_{2}\right|$, then $k$ and $m$ are all positive integers and it follows from (44) that

$$
M\left[\phi_{1} r^{k+2 n} e^{-r^{2}}\right](2|\alpha|) M\left[\phi_{2} r^{m+2 n} e^{-r^{2}}\right](2|\alpha|)=0
$$

for all $\alpha \gtrsim \alpha_{0}$. It follows that the function

$$
F(z):=M\left[\phi_{1} r^{k+2 n} e^{-r^{2}}\right](2 z) M\left[\phi_{2} r^{m+2 n} e^{-r^{2}}\right](2 z)
$$

vanishes on $\left|\alpha_{0}\right|,\left|\alpha_{0}\right|+1, \cdots$. Thus the function

$$
\widetilde{F}(z):=F\left(z+\left|\alpha_{0}\right|\right)=M\left[\phi_{1} r^{k+2 n+2\left|\alpha_{0}\right|} e^{-r^{2}}\right](2 z) M\left[\phi_{2} r^{m+2 n+2\left|\alpha_{0}\right|} e^{-r^{2}}\right](2 z)
$$

vanishes on $\mathbb{N}$. By Proposition $2.5 \widetilde{F}$ vanishes on the set $\{z \in \mathbb{C}: \operatorname{Re}(z)>0\}$. Since $\widetilde{F}$ is holomorphic we have that either $M\left[\phi_{1} r^{k+2 n+2\left|\alpha_{0}\right|} e^{-r^{2}}\right] \equiv 0$ or $M\left[\phi_{2} r^{m+2 n+2\left|\alpha_{0}\right|} e^{-r^{2}}\right] \equiv 0$, that is either $\phi_{1}$ or $\phi_{2}$ equals zero.

Theorem 6.2. Let $p, s$ be multi-indices and let $\phi, \psi$ be radial functions in $\operatorname{Sym}_{>0}\left(\mathbb{C}^{n}\right)$. If $\left[T_{\psi}, T_{\xi^{p} \bar{\xi}^{s}}\right]$ has finite rank then the rank is equal to zero.

Proof By Lemmas 3.1 and 5.5 we have that

$$
T_{\psi} T_{\xi^{p}} \bar{\xi}^{s} \phi^{\alpha}= \begin{cases}\frac{2(p+\alpha) ! M\left[\phi e^{-r^{2}}\right](2|\alpha|+|p|-|s|+2 n)}{(n+|p+\alpha|-1) !(p+\alpha-s) !} \frac{M\left[\psi e^{-r^{2}}\right](2|\alpha+p-s|+2 n)}{(n+|\alpha+p-s|-1) !} z^{\alpha+p-s}, & \text { if } p+\alpha \gtrsim s \\ \frac{2 s ! M\left[\phi e^{-r^{2}}\right](|s|-|p|+2 n) M\left[\psi e^{-r^{2}}\right](2|s-p-\alpha|+2 n)}{(s-p-\alpha) !(n+|s|-1) !(n-|s-p-\alpha|-1) !} \bar{z}^{s-p-\alpha}, & \text { if } p+\alpha \lesssim s \\ 0, & \text { otherwise, }\end{cases}
$$

and

$$
T_{\xi^{p} \bar{\xi}^{s} \phi} T_{\psi} z^{\alpha}= \begin{cases}\frac{2 M\left[\psi e^{-r^{2}}\right](2|\alpha|+2 n)}{(n+|\alpha|-1) !} \frac{!(p+\alpha) ! M\left[\phi e^{-r^{2}}\right](2|\alpha|+|p|-|s|+2 n)}{(n+|p+\alpha|-1) !(p+\alpha-s) !} z^{\alpha+p-s}, & \text { if } \alpha+p \gtrsim s \\ \frac{2 s ! M\left[\phi e^{-r^{2}}\right](|s|-|p|+2 n) M\left[\psi e^{-r^{2}}\right](2|\alpha|+2 n)}{(s-p-\alpha) !(n+|s|-1) !(n-|\alpha|-1) !} \bar{z}^{s-p-\alpha}, & \text { if } p+\alpha \lesssim s \\ 0, & \text { otherwise. }\end{cases}
$$

Let $S=\left[T_{\psi}, T_{\xi^{p} \bar{\xi} \phi}\right]$. Then for $\alpha+p \gtrsim s$ we have that

$$
\begin{aligned}
S\left(z^{\alpha}\right) & =\frac{2(p+\alpha) ! z^{\alpha+p-s}}{(n+|p+\alpha|-1) !(p+\alpha-s) !} \\
& \times \frac{M\left[\phi e^{-r^{2}}\right](2|\alpha|+|p|-|s|+2 n) M\left[\psi e^{-r^{2}}\right](2|\alpha+p-s|+2 n)}{(n+|\alpha+p-s|-1) !} \\
& -\frac{M\left[\psi e^{-r^{2}}\right](2|\alpha|+2 n) M\left[\phi e^{-r^{2}}\right](2|\alpha|+|p|-|s|+2 n)}{(n+|\alpha|-1) !}
\end{aligned}
$$


and for $p+\alpha \lesssim s$,

$$
\begin{aligned}
S\left(z^{\alpha}\right) & =\frac{2 s ! \bar{z}^{s-p-\alpha}}{(n+|s|-1) !(s-p-\alpha) !} \\
& \times \frac{M\left[\phi e^{-r^{2}}\right](|s|-|p|+2 n) M\left[\psi e^{-r^{2}}\right](2|s-\alpha-p|+2 n)}{(n+|s-\alpha-p|-1) !} \\
& -\frac{M\left[\psi e^{-r^{2}}\right](2|\alpha|+2 n) M\left[\phi e^{-r^{2}}\right](2|s|-|p| \mid+2 n)}{(n+|\alpha|-1) !},
\end{aligned}
$$

and $S\left(z^{\alpha}\right)=0$ if $\alpha$ does not satisfies $\alpha+p \gtrsim s, p+\alpha \lesssim s$. Since $\left\{S\left(z^{\alpha}\right)\right\}_{\alpha \in \mathbb{N}^{n}}$ has finite rank, there exists $\alpha_{0}$ such that $p+\alpha_{0} \gtrsim s$ and $S\left(z^{\alpha}\right)=0$ for all $\alpha \gtrsim \alpha_{0}$. Thus if $\alpha \gtrsim \alpha_{0}$ and $p+\alpha \gtrsim s$ then the function

$$
\Phi(z):=M\left[\phi r^{2 n} e^{-r^{2}}\right](2 z+k)\left[\frac{M\left[\psi r^{2 n} e^{-r^{2}}\right](2 z)}{\Gamma(z)}-\frac{M\left[\psi r^{2 n} e^{-r^{2}}\right](2 z+2 k)}{\Gamma(z+k)}\right],
$$

where $k=|p|-|s|$, is holomorphic on $\operatorname{Rez}>\max (-1,-k-1)$ and vanishes on $\left\{\left|\alpha_{0}\right|,\left|\alpha_{0}\right|+1, \cdots\right\}$. From the choice of $\alpha_{0}$, we get that $k \geq 0$. It follows by Corollary 2.6 that the function $\Phi(z)=0$ for all $z$ with $\operatorname{Rez}>\max (-1,-k-1)$. That is, $S\left(z^{\alpha}\right)=0$ for all $\alpha \in \mathbb{N}^{n}$. In a similar manner, we show that $S\left(\bar{z}^{\alpha}\right)=0$ for all $\alpha \in \mathbb{N}^{n}$. Hence $S$ is of rank zero.

It is easy to see that the methods of the proofs for Theorems 6.1 and 6.2 can be applied to the case of Fock Toeplitz operators.

\section{References}

Agbor, D., \& Bauer, W. (2014). Heat flow and an algebra of Toeplitz operators on the Segal-Bargmann Space. Integr. Equ. and Oper. Theory, 81, 271-299. https://doi.org/10.1007/s00020-014-2205-2

Aleman, A., \& Vukotic, D. (2009). Zero products of Toeplitz operators. Duke Math. J., 148(3), 373-403. https://doi.org/10.1215/00127094-2009-029

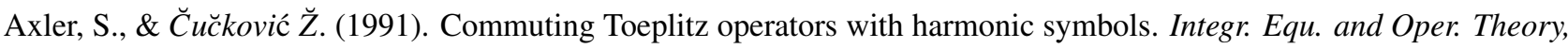
14, 1-12. https://doi.org/10.1007/BF01194925

Bauer, W. (2009). Berezin-Toeplitz quantization and composition formulas. J. Funct. Anal., 256, 3107-3142. https://doi.org/10.1016/j.jfa.2008.10.002

Bauer, W., \& Le, T. (2011). Algebraic properties and the finite rank problem for Toeplitz operators on the Segal-Bargmann space. J. Funct. Anal. 261(9), 2617-2640. https://doi.org/10.1016/j.jfa.2011.07.006

Bauer, W., \& Issa, H. (2012). Commuting Toeplitz operators with quasi-homogeneous symbols on the Segal-Bargmann Space. J. Math. Anal. Appl., 386, 213-235. https://doi.org/10.1016/j.jmaa.2011.07.058

Bauer, W., \& Lee, L. J. (2011). Commuting Toeplitz operators on the Segal- Bargmann Space. J. Funct. Anal., 260, 460-489. https://doi.org/10.1016/j.jfa.2010.09.007

Bauer, W., Choe, B. R., \& Koo, H. (2015). Commuting Toeplitz operators with Pluri harmonic symbols. J. Funct. Anal., 268, 3017-3060. https://doi.org/10.1016/j.jfa.2015.03.003

Brown, A., \& Halmos, P. R. (1964). Algebraic properties of Toeplitz operators Journal fr die reine und angewandte Mathematik, 213, 89-102.

Choe, B. R., \& Koo, H. (1993). Pluriharmonic symbols of commuting Toeplitz operators. Illinois J. Math. 37, 424-436.

Choe, B. R., Lee, J. Nam, K., \& Zheng, D. (2007). Products of Bergman space Toeplitz operators on the polydisk. Mathematische Annalen, 337(2), 295-316.https://doi.org/10.1007/s00208-006-0034-6

Coburn, L. A. (2007). On the Berezin-Toeplitz calculus. Proc. Amer. Math. Soc. 129(11), 3331-3338. https://doi.org/10.1090/S0002-9939-01-05917-2

$\breve{C}$ učković, $\breve{Z}$., \& Rao, N. V. (1998). Mellin transform, monomial symbols, and commuting Toeplitz operators. J. Funct. Anal., 154(1), 195-214. https://doi.org/10.1006/jfan.1997.3204

Dong, X. T., \& Zhou, Z. H. (2009). Algebraic properties of Toeplitz operators with radial symbols on the Bergman space of the unit ball. Integr. Equ. and Oper. Theory 64(1), 137-154. https://doi.org/10.1007/s00020-009-1677-y

Englis, M. (2009). Berezin transforms on the Pluriharmonic Bergman spaces. Trans. Amer. Math. Soc. 361(3), 11731188. https://doi.org/10.1090/S0002-9947-08-04653-9 
Folland, L. B. (1989). Harmonic Analysis in Phase Space. Princeton University Press, Princeton, New Jersey, 1989.

Guan, H., Liu, L., \& Lu, Y. (2013). Algebraic properties of Quasihomogeneous and separately Quasihomogeneous Toeplitz operators on the pluriharmonic Bergman space. Journal of Function spaces and Applications, 2013, Article ID 252037, 12 pages.

Luecking, D. (2008). Finite rank Toeplitz operators on the Bergman space. Proc. Amer. Math. Soc, 136(5), 1717-1723. https://doi.org/10.1090/S0002-9939-07-09119-8

Rozenblum, G., \& Shirokov, N. (2010). Finite rank Bergman-Toeplitz and Bargmann-Toeplitz operators in many dimensions. Complex Anal. Oper. Theory, 4(4), 767-775. https://doi.org/10.1007/s11785-009-0006-4

Yang ,J., Liu, L., \& Lu, Y. (2013). Algebraic properties of Toeplitz operators on the pluriharmonic Bergman space. Journal of Function spaces and Applications, 2013, Article ID 578436, 12 pages.

\section{Copyrights}

Copyright for this article is retained by the author(s), with first publication rights granted to the journal.

This is an open-access article distributed under the terms and conditions of the Creative Commons Attribution license (http://creativecommons.org/licenses/by/4.0/). 